\title{
A NON-CONTROLLED STUDY OF A MULTI-FACTORIAL EXERCISE AND NUTRITIONAL INTERVENTION TO IMPROVE FUNCTIONAL PERFORMANCE AND PREVENT FRAILTY PROGRESSION IN COMMUNITY-DWELLING PRE-FRAIL OLDER ADULTS
}

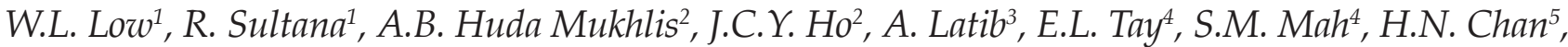 \\ Y.S. $N g^{6}$, L. Tay
}

\begin{abstract}
Background: Preventing frailty is important to avoid adverse health outcomes. Intervention studies have largely focused on frail elderly, although the intermediate pre-frail state may be more amenable to improvement. Objectives: This study aims to assess how physical performance may change among pre-frail elderly enrolled in a pragmatic non-controlled exercise and nutritional intervention programme. Methods: This is a non-controlled study involving a 4-month exercise and nutritional intervention for community dwelling pre-frail older adults. Pre-frailty was defined as the presence of 1 or 2 positive responses on the FRAIL questionnaire, or evidence of weak grip strength ( $<26 \mathrm{~kg}$ for males; $<18 \mathrm{~kg}$ for females) or slow gait speed $(<0.8 \mathrm{~m} / \mathrm{s})$ amongst participants who were asymptomatic on FRAIL. Physical performance in flexibility, grip and lower limb strength, endurance, balance, and Short Physical Performance Battery were measured at 3 time-points: baseline, 3-month from recruitment (without intervention), and immediate post-intervention. Repeated measures mixed model analysis was performed to compare physical performance measures across the 3 time-points. Results: 94 pre-frail participants were eligible for intervention, of whom 59 (mean age $=70.9 \pm 7.2$ years) were ready for the post-intervention review. $21(35.6 \%)$ transitioned to robust phenotype while $32(54.2 \%)$ remained as pre-frail. Significant improvement post-intervention was observed in lower limb strength and power, evident on reduction in time taken for 5 sit-to-stand repetitions $(0.46 \pm 0.20 \mathrm{~s}, \mathrm{p}=0.03)$. There was no significant change to the other physical performance measures examined. Conclusion: We observed reversibility of pre-frailty, and the benefit of multi-component intervention in improving physical performance of pre-frail older adults. The findings in this non-controlled study will need to be corroborated with future controlled trials.
\end{abstract}

Key words: Pre-frailty, multi-factorial intervention, exercise, nutrition.

\section{Introduction}

Pre-frailty is generally regarded as the intermediate stage between being robust and frail (1), and almost 6-times more prevalent than frailty amongst communitydwelling older persons (2). Although considerably less vulnerable and more likely to revert to robustness than their frail counterparts (3), pre-frail elderly present higher risk than their robust peers for adverse health events such as falls, hospitalization and disability (4).

The importance of physical activity and nutrition as strategies for preventing or delaying frailty in older adults has been established (5) and included in

\footnotetext{
1. Duke-NUS Medical School, Singapore; 2. Research Office, Sengkang General Hospital, Singapore; 3. Health Services Research and Evaluation, SingHealth, Singapore; 4. Physiotherapy Department, Sengkang General Hospital, Singapore; 5. Department of Dietetics, Sengkang General Hospital, Singapore; 6. Department of General Medicine, Sengkang General Hospital, Singapore
}

Corresponding Author: Ms. Low Wan Li, Duke-NUS Medical School, 8 College Road, Singapore 169857, Email: e0178630@u.duke.nus.edu

Received October 10, 2019

Accepted for publication December 8, 2019 guidelines for frailty management (6). While earlier intervention studies have largely focused on frail older adults, a systematic review targeting older people with mild or pre-frailty reported mixed effects of exercise as a single-domain intervention (7). Multidomain interventions were reportedly more effective than mono-domain interventions in improving frailty status and physical function in frail and pre-frail older adults, particularly for the combination of exercise and nutritional intervention (8). However, studies involving dietary management have typically relied on a range of nutritional supplements focused on single nutrient or food groups such as protein and vitamin D with inconsistent effects that were more likely to benefit those who were malnourished or severely frail [9]. Further, compliance and sustained provision of such supplements beyond the study period remain major limiting factors. Thus, the positive change in dietary intake with accompanying improvement in frailty and depression scores observed with individualized nutrition 
education in a predominantly pre-frail cohort suggests that differential nutritional approaches may be warranted across the frailty spectrum (10).

This non-controlled study aims to assess how functional fitness and frailty status may change among community-dwelling pre-frail older adults in a combined physical exercise and nutrition programme. However, contrary to earlier studies, the nutritional intervention focused on tailoring intake and dietary habits of our local elderly to achieve guideline recommendations without the use of nutritional supplements.

\section{Methods}

\section{Study Design and Participants}

Potential participants were identified through our ongoing community frailty screening "Individual Physical Proficiency Test for Seniors (IPPT-S)", which had been previously described (1). Briefly, the screening platform was rotated around the void decks of public housing blocks, senior activity centres and community clubs in the North-Eastern region of Singapore. Eligible participants (aged $>55$ years and can ambulate independently) in IPPT-S completed a multi-domain geriatric screen that included assessment of mood (Geriatric Depression Scale, GDS) (11), cognition (modified version of Chinese Mini Mental State Examination, CMMSE) (12), nutrition (Mini Nutritional Assessment-Short Form, MNA-SF) (13), and functional performance (Barthel's Index for activities of daily living (ADL) and Lawton and Brody's instrumental ADL) (14, 15). Frailty status was assessed using the FRAIL scale, a simple questionnaire comprising 5 components - Fatigue, Resistance, Ambulation, Illnesses, and Loss of weight. 1 point was assigned for each component, with scores 0 indicating robust, 1-2 pre-frail and 3-5 frail respectively (16). The physical fitness test battery modified from the Senior Fitness Test (17) included measures of gait speed, grip strength, upper and lower limb flexibility, upper limb dexterity, lower limb strength and power, balance and cardiorespiratory endurance. Operational definition for pre-frailty to be eligible for intervention was based on (i) 1 or 2 positive responses on the FRAIL questionnaire or (ii) 0 positive response on FRAIL but with weak grip strength $(<26 \mathrm{~kg}$ for males; $<18 \mathrm{~kg}$ for females) or slow gait speed $(<0.8 \mathrm{~m} / \mathrm{s})$ based on the Asian Working Group for Sarcopenia (18). Ethics approval was obtained from Singhealth Institutional Review Board and all participants provided written informed consent. This study is registered with ClinicalTrials.gov (Identifier: NCT04656938).

\section{Intervention}

This is an ongoing study. All IPPT-S participants were invited to attend 4 general group education sessions on frailty prevention that were scheduled during the 3 months after each screening cycle. Eligible pre-frail participants were subsequently enrolled in a 4-month multi-disciplinary intervention programme comprising (i) once-weekly group-based exercise classes lasting 1 hour each session (total of 16 sessions) with individually prescribed home exercises for maintenance between sessions and (ii) group-based nutritional education (6 sessions). Group size was maintained at 8-10 participants to ensure that each participant received adequate attention. While the intensity of exercise was not measured, the target was to achieve at least moderate intensity as tolerated by the seniors. The exercises focused on strength, balance and endurance training, with a warm-up and cool-down routine. TheraBands and step boards were used for resistance and balance training respectively. The exercise sessions were designed for progressive intensity (such as increasing number of repetitions, increased resistance of the TheraBands, height of step boards) based on the group's progress, while catering for individual variability, with group sessions conducted under the supervision of a physiotherapist and an exercise physiologist. Each session commenced with 5 minutes of dynamic warm-up e.g. slow marching with small to big arm circles, followed by 45 minutes of exercises focusing on: a) balance, coordination and speed e.g. heel to toe walks; b) strength e.g. rising from a chair and Theraband exercises for lower and upper limbs respectively; and c) endurance e.g. fast walking. All sessions ended with 5 minutes of static cool down e.g., stretching muscles of the thigh and arms with slow breathing. Individually prescribed structured home exercise folders comprising pictorials and written explanations were provided at the end of each session, to encourage participants to maintain regular physical exercise between the group classes. The nutritional intervention was delivered with the aim of facilitating healthy eating habits to achieve adequate protein, energy, calcium and Vitamin D through regular food and beverages that are more specific to the Asian palate. There were 6 sessions over the 4-month intervention period with 2 sessions per month in the first and second months and 1 session per month in the third and fourth months. Each session lasted 1.5 hours and was delivered by a trained nutritionist, incorporating a combined modality of teaching methods that included didactics, food-based games and grocery-shopping trips with sponsored vouchers to provide guidance on choosing quality foods within budget. Attendance at the exercise and nutrition classes was tracked, with minimum attendance set at 50\% of all scheduled sessions (19).

\section{Outcome Measures}

Outcome measures include frailty status based on the FRAIL scale and individual physical performance measures. Gait speed was measured based on time 
taken to walk $10 \mathrm{~m}$ at usual pace. Grip strength was measured using a JAMAR Plus Hand Dynamometer (Sammons Preston, Bolingbrook, IL, USA), following the Southampton protocol with participant seated and elbow at 90-degree flexion (20). 2 trials were performed for each hand, alternating sides during the test, and the maximal reading from all trials was used for analysis. Upper and lower limb flexibility was measured via back scratch (21) and modified sit-and-reach tests (22) respectively. In back scratch test, participants were asked to place 1 hand over a shoulder and the other up the middle of their back with the fingers extended. The distance (in $\mathrm{cm}$ ) in which the middle fingers of both hands overlapped with each other or failed to meet was recorded as positive and negative scores, respectively. In the modified sit-andreach test, participants sat on the edge of a chair with one leg extended before them and reached forward to touch their toes with their fingers. Likewise, the distance (in $\mathrm{cm}$ ) in which the extended third finger reached beyond the toe or failed to touch the toe was documented as positive and negative, respectively. The higher reading from 2 attempts in each test was used in the analysis. Upper limb dexterity was assessed via box-and-block test (23) where participants were instructed to briskly pick up blocks from one side of a box and place them on another side across a barrier. The number of blocks transferred within 1 minute was recorded. The higher reading taken from 2 trials for each arm was used for analysis. Lower limb strength and power was measured using the chair stand test, recording the duration taken to complete 5 chair-stands as well as the number of chair stands completed within 30 seconds (24). Balance was assessed via the Timed-Up-and-Go test (25) and semi-tandem and tandem stands. In the former, participants were requested to rise from a seated position, walk briskly round a cone that was placed $3 \mathrm{~m}$ away from their chair, return to the chair and resume a fully seated position. In the latter, we used the side-by-side, semi-tandem and full-tandem standing tests in the Short Physical Performance Battery (SPPB) (26). Cardiorespiratory endurance was measured via 6-minute walk test (27), using a 20-metre path with constant encouragement throughout the test. The distance traversed in 6 minutes was recorded and participants were allowed to rest at any time during the test. Each participant was scored on the Short Physical Performance Battery for a composite measure of physical performance, applying established cut-offs in individual tests of gait speed, balance and chair-stand (28). These outcome measures were performed at 3 time-points: baseline screening, 3-month post-screening ("Pre-intervention" immediately prior to commencement of intervention) and at the end of the 4-month intervention programme ("Postintervention").

\section{Statistical Analysis}

Continuous variables were presented as means \pm SD while categorical variables were presented as absolute frequencies and relative percentages. Repeated measures mixed model analysis was performed to examine the association of intervention with individual outcome measures of physical performance. Time as fixed, a random effect at the participants' level and a random slope on time were used in this mixed model. Timewise estimates of scale for the outcome measures were performed at three time-points namely, 'Baseline', 'Preintervention' and 'Post-intervention'. A separate analysis was performed using time as a continuous variable. The analysis was repeated adjusting for attendance in group exercise and nutrition education sessions, as these were anticipated confounders. We performed exploratory analysis to compare changes in physical performance measures (from "Baseline" to "Post-intervention") between (i) pre-frail participants with FRAIL score 0 but exhibiting weak grip/ slow gait vs FRAIL score 1-2 at baseline, and (ii) pre-frail participants who reverted to robustness versus those who remained pre-frail postintervention. Level of significance was defined as $p$ value $<0.05$ and all tests performed were two sided. All analyses were performed on SAS University Edition (SAS/STAT®, SAS Institute Inc, NC, USA).

\section{Results}

94 participants fulfilled operational criteria for prefrailty and consented to the intervention programme. As this is an ongoing study with a 3-month interval between screening and intervention initiation across different community sites, 59 participants were ready for the post-intervention review at time of analysis. The average attendance was $80.1 \pm 14.0 \%$ and $85.0 \pm 16.6 \%$ for group exercise and nutrition classes respectively. Mean age was $70.9 \pm 7.2$ years, with female predominance $(81.4 \%) .26(44 \%)$ were reportedly robust on FRAIL scale but exhibited weak grip and / or slow gait speed while 33 $(56 \%)$ participants had a FRAIL score of 1 or 2 points.

Post-intervention, of 59 participants, 21 (35.6\%) transitioned to being robust while $32(54.2 \%)$ remained pre-frail. $6(10.2 \%)$ participants did not attend the postintervention review and were excluded from the final analysis for physical performance measures (Figure 1). There was no significant difference in age and gender between the participants who were included versus those who were excluded from the final analysis (Table 1).

Participants who transitioned to being robust postintervention were significantly younger, compared with those who remained pre-frail $(p<0.001)$. Pre-frail participants who had weak grip and/ or slow gait but "asymptomatic" on FRAIL were significantly less likely to revert to robustness. Baseline mood, cognition and nutritional status were similar between participants who 
Table 1

Baseline characteristics of overall cohort

\begin{tabular}{|c|c|c|c|}
\hline Baseline Characteristics & Included for Analysis (n=53) & Excluded for Analysis $(n=6)$ & P-value \\
\hline \multicolumn{4}{|l|}{ Eligibility criteria $(\mathrm{n}, \%)$} \\
\hline FRAIL 0 (weak grip / slow gait speed) & $24,45.3$ & $2,33.3$ & 0.58 \\
\hline FRAIL 1-2 & $29,54.7$ & $4,66.7$ & \\
\hline Age $($ mean \pm SD) & $70.8 \pm 7.0$ & $71.3 \pm 8.8$ & 0.87 \\
\hline Female $(\mathrm{n}, \%)$ & $44,83.0$ & $4,66.7$ & 0.33 \\
\hline Chinese $(\mathrm{n}, \%)$ & $42,79.0$ & $5,83.3$ & 0.92 \\
\hline BMI, $\mathrm{kg} / \mathrm{m}^{2}($ mean $\pm \mathrm{SD})$ & $25.0 \pm 5.4$ & $21.5 \pm 5.4$ & 0.14 \\
\hline \multicolumn{4}{|l|}{ MNA-SF status (n, \%) } \\
\hline Normal & $39,73.6$ & $4,66.7$ & 0.72 \\
\hline At risk / Malnourished & $14,26.4$ & $2,33.3$ & \\
\hline CMMSE score (mean \pm SD) & $24.6 \pm 2.5$ & $24.3 \pm 3.1$ & 0.78 \\
\hline GDS score $($ mean $\pm \mathrm{SD})$ & $2.9 \pm 2.6$ & $2.0 \pm 2.2$ & 0.44 \\
\hline$\%$ Depressed, GDS score $\geq 5(n, \%)$ & $10,18.9$ & $1,16.7$ & 0.90 \\
\hline ADL score $($ mean $\pm \mathrm{SD})$ & $19.7 \pm 0.7$ & $19.8 \pm 0.41$ & 0.66 \\
\hline IADL score (mean $\pm \mathrm{SD})$ & $21.6 \pm 2.2$ & $21.3 \pm 4.1$ & 0.78 \\
\hline \multicolumn{4}{|l|}{ Attendance (mean \pm SD) } \\
\hline Group exercise class & $80.7 \pm 13.7$ & $75.0 \pm 16.3$ & 0.35 \\
\hline Nutrition class & $85.5 \pm 16.4$ & $80.6 \pm 19.5$ & 0.49 \\
\hline
\end{tabular}

Figure 1

Flow chart of study model

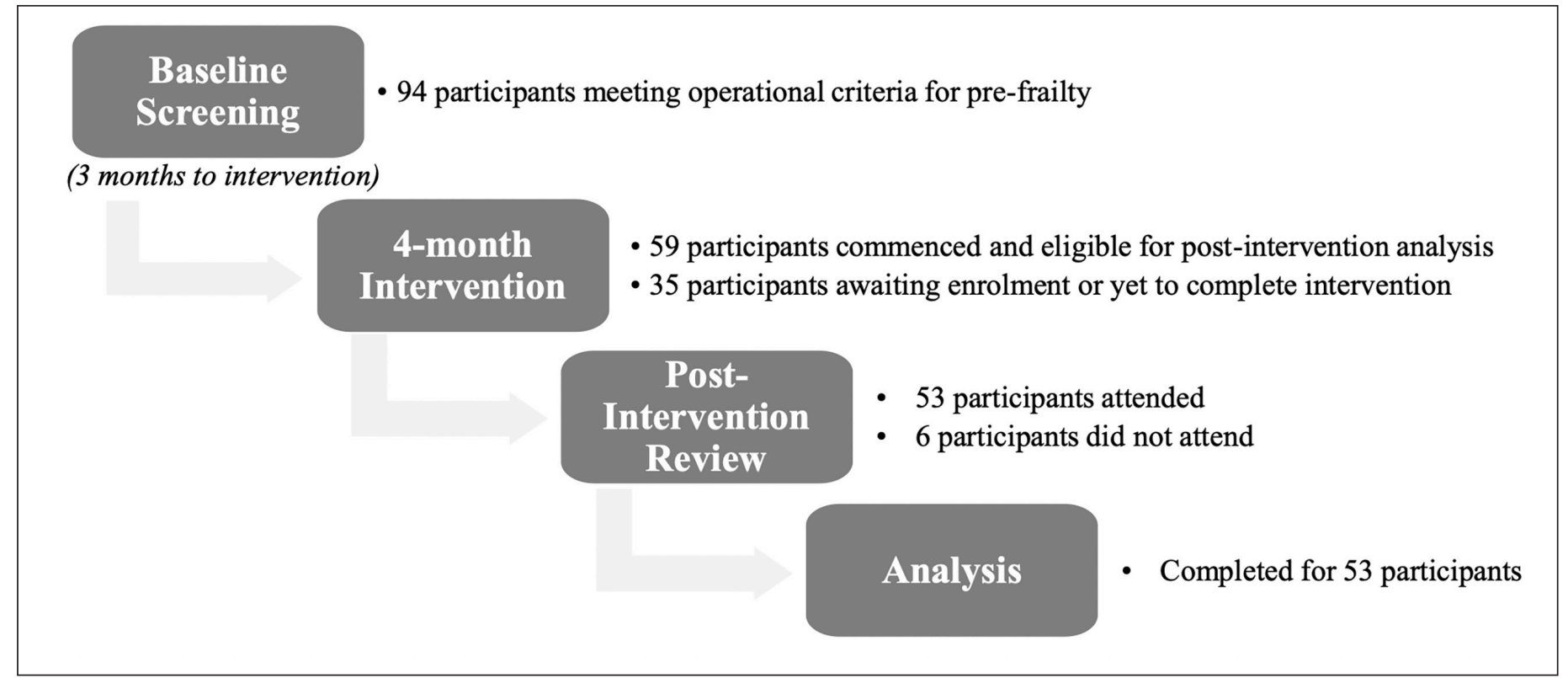

reverted and those who remained pre-frail. There was no difference in attendance at exercise and nutritional classes between participants who reverted to robustness and those who remained pre-frail (Table 2).

Lower limb strength represented by time taken to complete 5 chair-stands improved significantly, with mean reduction of $0.46 \pm 0.20$ s per time-point $(p=0.03)$. We observed a trend for improved grip strength and cardiorespiratory endurance on the 6-minute walk-test, albeit not achieving statistical significance. There was no change to overall physical performance on SPPB, upper limb dexterity and flexibility measures (Table 3). Repeating the analyses with adjustment for adherence rates yielded similar results.

Exploratory analysis was performed comparing participants who reverted to robustness versus those who remained pre-frail at post-intervention for all physical performance measures. We observed significantly 
Table 2

Baseline characteristics by pre-frailty reversal at end of intervention

\begin{tabular}{|c|c|c|c|}
\hline \multirow[t]{2}{*}{ Baseline Characteristics } & \multicolumn{3}{|c|}{ Post-Intervention } \\
\hline & Robust (n=21) & Pre-frail $(n=32)$ & P-value \\
\hline \multicolumn{4}{|l|}{ Eligibility criteria $(\mathrm{n}, \%)$} \\
\hline FRAIL 0 (weak grip / slow gait speed) & $5,23.8$ & $19,59.4$ & 0.01 \\
\hline FRAIL 1-2 & $16,76.3$ & $13,40.6$ & \\
\hline Age, years $($ mean $\pm S D)$ & $66.4 \pm 4.2$ & $73.7 \pm 7.0$ & $<0.01$ \\
\hline Female $(\mathrm{n}, \%)$ & $18,85.7$ & $26,81.3$ & 0.67 \\
\hline Chinese $(\mathrm{n}, \%)$ & $17,81.0$ & $25,78.1$ & 0.86 \\
\hline $\mathrm{BMI}, \mathrm{kg} / \mathrm{m} 2($ mean $\pm \mathrm{SD})$ & $24.4 \pm 4.3$ & $25.4 \pm 6.1$ & 0.53 \\
\hline \multicolumn{4}{|l|}{ MNA-SF status $(\mathrm{n}, \%)$} \\
\hline Normal & $16,76.2$ & $23,71.9$ & 0.73 \\
\hline At risk / Malnourished & $5,23.8$ & $9,28.1$ & \\
\hline CMMSE score (mean \pm SD) & $25.2 \pm 2.6$ & $24.3 \pm 2.5$ & 0.20 \\
\hline GDS score $($ mean $\pm \mathrm{SD})$ & $2.2 \pm 2.6$ & $3.2 \pm 2.5$ & 0.13 \\
\hline$\%$ Depressed, GDS score $\geq 5(\mathrm{n}, \%)$ & $4,19.0$ & $6,18.8$ & 0.98 \\
\hline ADL score $($ mean $\pm S D)$ & $19.9 \pm 0.4$ & $19.6 \pm 0.8$ & 0.09 \\
\hline IADL score (mean \pm SD) & $22.2 \pm 1.0$ & $21.3 \pm 2.7$ & 0.13 \\
\hline \multicolumn{4}{|l|}{ Attendance $($ mean \pm SD) } \\
\hline Group exercise class & $82.2 \pm 13.2$ & $79.7 \pm 14.2$ & 0.53 \\
\hline Nutrition class & $84.1 \pm 17.9$ & $86.5 \pm 15.5$ & 0.62 \\
\hline
\end{tabular}

greater gain in grip strength in the group demonstrating reversal to robustness compared with their counterparts who remained pre-frail $[+1.95 \mathrm{~kg}(95 \% \mathrm{CI}: 0.57,3.33)$ vs $-0.02 \mathrm{~kg}(95 \% \mathrm{CI}:-0.96,0.91)$, mean difference: 1.98 $\mathrm{kg}(95 \%$ CI: 0.35, 3.61), p=0.02]. Between pre-frailty definitions, greater improvement in 6-minute walk test was observed post-intervention in the group with FRAIL score 1-2 compared with "asymptomatic" (FRAIL score $0)$ but with objective weak grip/ slow gait $[+59.66 \mathrm{~m}$ $(95 \%$ CI $21.87,97.45)$ vs $+9.16 m(95 \%$ CI -19.11, 37.42), mean difference: $50.50 \mathrm{~m}$ (95\% CI: 4.37, 96.63), $\mathrm{p}=0.03]$. There was no significant difference in the other physical performance measures.

\section{Discussion}

Our single-arm study builds on the current literature on frailty intervention but focusing specifically on prefrail older individuals. Over one-third of pre-frail seniors reverted to being robust, with improvements in strength and endurance following a multi-component exercise and nutritional intervention programme.

A recent systematic review of frailty state transitions in community-dwelling older adults suggested that frailty progression (worsening) was more likely to occur than frailty regression (improvement), and transitions between extreme states (frail to robust and vice versa) were rare. Significantly, approximately one-quarter of pre-frail seniors transitioned to being frail or mortality (29). An observational study had also noted half of pre-frail older adults remained in the pre-frail state over 2 years, with about one quarter reverting to robustness (30). Thus, the intermediate pre-frail state offers an optimal window of opportunity for intervention to avoid worsening frailty and adverse outcomes, with approximately one-third of our participants reverting from pre-frailty to robustness. However, this is much lower than the observed $80 \%$ reversal from pre-frailty to robust phenotype in a recent study in Hong Kong involving a multi-component frailty prevention programme, which included emphasis on cognitive training as well as socialization through board game activities (31). With frailty being multifactorial, interventions that address all key components contributing to the frailty syndrome maybe necessary. This is in line with another study conducted on a group of rural elderly participants in Korea, where the SPPB score is noted to have increased by 3.18 points from baseline after undergoing a 6-month intervention comprising group exercise, nutrition, depression management, deprescribing medication and home hazard reduction (32). Our earlier study had supported pre-frailty as an intermediate state between being robust and frail, and associated with depression, malnutrition, sarcopenia and socio-economic status (1).

The observed improvement in lower limb strength is consistent with the Hong Kong study which demonstrated improved physical performance and frailty status amongst pre-frail elderly enrolled in a multi-component frailty prevention programme (31). We 
Table 3

Changes in Physical Performance Measures

\begin{tabular}{|c|c|c|c|c|}
\hline & $\begin{array}{c}\text { Baseline: } \\
\text { Mean }(95 \% \mathrm{CI})\end{array}$ & $\begin{array}{l}\text { Pre-Intervention: } \\
\text { Mean }(95 \% \mathrm{CI})\end{array}$ & $\begin{array}{c}\text { Post-Intervention: } \\
\text { Mean }(95 \% \text { CI) }\end{array}$ & $\begin{array}{c}\text { B-Coefficient }{ }^{1} \text { : } \\
\text { Mean (SD, p-value) }\end{array}$ \\
\hline \multicolumn{5}{|c|}{ Composite Measure } \\
\hline SPPB & $10.61(10.11,11.11)$ & $10.78(10.27,11.28)$ & $11.06(10.55,11.57)$ & $+0.14(0.09), \mathrm{p}=0.12$ \\
\hline \multicolumn{5}{|c|}{ Gait Performance } \\
\hline GS $(\mathrm{m} / \mathrm{s})$ & $1.13(1.06,1.20)$ & $1.18(1.11,1.25)$ & $1.19(1.12,1.26)$ & $+0.01(0.01), \mathrm{p}=0.32$ \\
\hline TUG (s) & $11.36(10.45,12.26)$ & $11.01(10.10,11.92)$ & $11.30(10.38,12.23)$ & $+0.08(0.17), \mathrm{p}=0.63$ \\
\hline \multicolumn{5}{|c|}{ Lower Limb Strength and Power } \\
\hline STS & $13.97(12.65,15.28)$ & $15.19(13.87,16.51)$ & $14.67(13.34,16.00)$ & $+0.13(0.22), \mathrm{p}=0.56$ \\
\hline 5STS (s) & $11.92(10.82,13.02)$ & $10.79(9.68,11.90)$ & $10.28(9.15,11.41)$ & $-0.46(0.20), p=0.03$ \\
\hline \multicolumn{5}{|c|}{ Upper Limb Strength and Dexterity } \\
\hline GST (kg) & $19.32(18.08,20.55)$ & $19.83(18.59,21.07)$ & $20.10(18.85,21.35)$ & $+0.26(0.14), \mathrm{p}=0.08$ \\
\hline BBT & $49.32(46.76,51.88)$ & $50.69(48.10,53.27)$ & $49.51(46.87,52.14)$ & $-0.23(0.45), \mathrm{p}=0.61$ \\
\hline \multicolumn{5}{|l|}{ Flexibility } \\
\hline MSRT (cm) & $-3.08(-5.86,-0.30)$ & $-5.37(-8.19,-2.55)$ & $-3.90(-6.79,-1.01)$ & $-0.11(0.64), p=0.86$ \\
\hline BST (cm) & $-7.55(-10.82,-4.28)$ & $-7.41(-10.68,-4.14)$ & $-8.07(-11.35,-4.78)$ & $-0.08(0.26), p=0.76$ \\
\hline \multicolumn{5}{|c|}{ Cardiorespiratory Endurance } \\
\hline 6MWT $(\mathrm{m})$ & $386.81(357.3,416.3)$ & $418.23(388.5,448.0)$ & $421.76(391.8,451.7)$ & $+7.20(4.10), p=0.08$ \\
\hline
\end{tabular}

also observed significantly greater improvement in grip strength amongst pre-frail participants who reverted to robustness post-intervention. With frailty and weakness contributing to falls risk in older adults $(33,34)$, the improvement in lower limb strength and power may potentially prevent adverse outcomes associated with falls in frail elderly. In addition, in a recent review that studied the association between lifestyle interventions and healthy aging as defined by intrinsic capacity, it was found that multi-domain intervention was associated with improvements in locomotion by way of performance-based test of lower limb function (35).

While the observed improvements in cardiorespiratory endurance and grip strength did not meet statistical significance, earlier studies suggested pre-frail elderly are likely to be functioning at the limit of their capacity to fulfil activities of daily living (36), and improvements in 6MWT and grip strength in the early months post-discharge had a positive impact on prognosis amongst frail hospitalized elderly (37). Specifically, our exploratory analysis supported significantly greater gains in cardiorespiratory endurance amongst pre-frail participants with symptoms on the FRAIL scale compared with their counterparts who were "asymptomatic" but had objective weak grip or slow gait. The latter were also less likely to revert to being robust. Further research should examine whether differential intervention approaches may be warranted once objective declines in physical performance set in, such as intensity of exercise training and intervention duration. The lack of gains in gait speed and balance may be attributed to the focus on resistance and cardiorespiratory endurance exercises both in the group sessions and at home, though step-boards were employed for balance training at the weekly sessions. Another reason could be the shorter duration of our intervention programme, over a 4-month period as opposed to other multi-factorial intervention that span over 6 months (32).

While we sought to address malnutrition through enforcing positive changes in dietary intake, more intensive approach including specific supplements may be necessary for those who are already malnourished. This echoes the findings of a previous study that suggests that frail elderly who are more compromised on nutrition status, may benefit more from nutritional supplementation (9). We observed $16(27 \%)$ of our cohort to be at-risk of malnutrition or malnourished at baseline on the Mini Nutrition Assessment-Short Form questionnaire, although this was not repeated immediately post-intervention.

We acknowledge several limitations. While adherence rate to intervention is adjusted, the lack of a control group limits the inference of association between the contribution by the multi-factorial intervention and the observed therapeutic effect. Further, with a small sample size and multiple outcome measures being analysed, the possibility of the significant improvement in lower limb strength arising from chance and a type 1 error cannot be dismissed. In conclusion, this multi-factorial intervention comprising physical exercise therapy and nutrition 
education sessions showed positive change in functional performance and possibly reversing frailty progression in pre-frail community-dwelling older adults. Longitudinal follow-up is ongoing to examine the sustainability of improvements in physical performance beyond the immediate period post-intervention. The findings in this non-controlled study will need to be corroborated with future controlled trials.

Acknowledgements: We thank the study participants, staff of the Senior Activity Centres and Resident Committees in Northeast Singapore for their logistical and manpower support.

Funding: This study is funded by National Medical Research Council Centre Grants (CGAug16C027 and CGAug16M011), National Innovation Challenge on Active and Confident Ageing (MOH/ NIC/HAIG04/2017), and AM-ETHOS Duke-NUS Medical Student Fellowship (AM-ETHOS01/FY2019/06-A06). The grants funded the research staff, assessment and exercise equipment, and on-site conduct of the trial.

Role of Sponsor: None.

Conflicts of interest: The authors have no conflicts of interest to declare, financial or otherwise.

Ethical standards: The authors declare that the study procedures adhere to all ethical standards. Ethics approval for this study was obtained from Singhealth Instituitional Review Board.

\section{References}

1. Lim YJ, Ng YS, Sultana R, et al. Frailty assessment in community dwelling older adults: a comparison of 3 diagnostic instruments. J Nutr Health Aging 2020;24(6):582-590.

2. Merchant RA, Chen MZ, Tan LWL, et al. Singapore Healthy Older People Everyday (HOPE) Study: Prevalence of Frailty and Associated Factors in Older Adults. JAMDA 2017;18(8):734e9-e14.

3. Gill TM, Gahbauer EA, Allore HG, Han L. Transitions between frailty states among community-living older persons. Arch Intern Med 2006;166:418-423.

4. Fried LP, Tangen CM, Walston J, et al. Frailty in older adults: evidence for a phenotype. Journal of Gerontology 2001;56A(3):M146-M156.

5. Kelaiditi E, Abellan van Kan G, Cesari M. Frailty: Role of Nutrition and Exercise. Curr Opin Clin Nutr Metab Care 2014;17(1):32-9.

6. Dent E, Lian C, Lim WS, et al. The Asia Pacific Clinical practice guidelines for the management of frailty. JAMDA 2017;17:564-575

7. Frost R, Belk C, Jovicic A, et al. Health promotion interventions for community-dwelling older people with mild or prefrailty: a systematic review and meta-analysis. BMC Geriatrics 2017;17(157).

8. Dedyne L, Deschodt M, Verschueren S, Tournoy J, Gielen E. Effects of multidomain interventions in (pre)frail elderly on frailty, functional and cognitive status: a systematic review. Clinical Interventions in Aging 2017;12:873-896.

9. Ng TP, Feng L, Nyunt MSZ, et al. Nutritional, physical, cognitive and combination interventions and frailty reversal among older adults: a randomized controlled trial. The American Journal of Medicine 2015;128(11):1226-1236.

10. Wu SY, Hsu LL, Hsu CC, et al. Dietary education with customised dishware and food supplements can reduce frailty and improve mental well-being in elderly people: a single-blind randomized controlled study. Asia Pac J Clin Nutr 2018;27(5):1018-1030.

11. Sheikh JI, Yesavage JA. 9/Geriatric Depression Scale (GDS). Clinical Gerontologist 1986;5(1-2):165-173.

12. Feng L, Chong MS, Lim WS, et al. The Modified Mini-Mental State Examination test: normative data for Singapore Chinese older adults and its performance in detecting early cognitive impairment. Singapore Med 2012;53(7):458.
13. Kaiser MJ, Bauer JM, Ramsch C, et al. Validation of the mini nutritiona assessment short-form (MNA-SF): A practical tool for identification of nutritional status. The Journal of Nutrition, Health \& Aging 2009;13(9):782-8:

14. Mahoney FI, Barthel D. Functional Evaluation: The Barthel Index. Maryland State Medical Journal 1965;14:61-65.

15. Lawton M.P, Brody EM. Assessment of Older People: Self-Maintaining and Instrumental Activities of Daily Living. The Gerontologist 1969;9(3):179-186.

16. Abellan van Kan G, Rolland Y, Bergman H, Morley JE, Kritchevsky SB, Vellas B. The I.A.N.A. Task Force on frailty assessment of older people in clinical practice. J Nutr Health Aging 2008;12(1):29-37.

17. Rikli RE, Jones CJ. Senior fitness test manual. 2001. Human Kinetics, Champaign, IL.

18. Chen LK, Liu LK, Woo J, et al. Sarcopenia in Asia: consensus report of the Asian working group for sarcopenia. JAMDA 2014;15:95-101.

19. Puts MTE, Toubasi S, Andrew MK, et al. Interventions to prevent or reduce the level of frailty in community-dwelling older adults: a scoping review of the literature and international policies. Age Ageing 2017;46(3):383-392.

20. Roberts HC, Denison HJ, Martin HJ, Patel HP, Syddall H, Cooper C, Sayer AA. A review of the measurement of grip strength in clinical and epidemiological studies: towards a standardised approach. Age Ageing 2011;40:423-9

21. Jones CJ, Rikli RE. Measuring functional fitness of older adults. J Active Aging 2002:24-30.

22. Jones CJ, Rikli RE, Max J, Noffal G. The reliability and validity of a chair sitand-reach test as a measure of hamstring flexibility in older adults. Res Q Exerc Sport 1998;69:338-43.

23. Desrosiers J, Bravo G, Hébert R, Dutil E, Mercier L. Validation of the Box and Block Test as a measure of dexterity of elderly people: reliability, validity, and norms studies. Arch Phys Med Rehabil 1994;75:751-5.

24. Glenn JM, Gray M, Binns A. Relationship of sit-to-stand lower-body powe with functional fitness measures among older adults with and without sarcopenia. J Geriatr Phys Ther 2017;40:42-50.

25. Podsiadlo D, Richardson S. The timed "Up \& Go": a test of basic and functional mobility for frail elderly persons. J Am Geriatr Soc 1991;39:142-8.

26. Guralnik JM, Simonsick EM, Ferrucci L, Glynn RJ, Berkman LF, Blazer DG, et al. A short physical performance battery assessing lower extremity function: association with self-reported disability and prediction of mortality and nursing home admission. J Gerontol 1994;49:M85-94.

27. ATS Committee on Proficiency Standards for Clinical Pulmonary Function Laboratories. ATS statement: guidelines for the six-minute walk test. Am J Respir Crit Care Med 2002;166:111-7.

28. Treacy D, Hassett L. The Short Physical Performance Battery. Journal of Physiotherapy 2018;64:61.

29. Kojima G, Taniguchi Y, Iliffe S, et al. Transitions between frailty states among community-dwelling older people: a systematic review and meta-analysis. Ageing Research Reviews 2019;50:81-88.

30. Lee JSW, Auyeung TW, Leung J, et al. Transitions in frailty states among community-living older adults and their associated factors. JAMDA 2014;15:281-286.

31. Yu R, Tong C, Ho F, Woo J. Effects of a multicomponent frailty prevention program in prefrail community dwelling older persons: a randomized controlled trial. JAMDA 2020;21:294.e1-10.

32. Jang IY, Jung HW, Park $\mathrm{H}$, et al. A multicomponent frailty intervention for socioeconomically vulnerable older adults: a design-delayed study. Clinical Interventions in Aging 2018;13:1799-1814.

33. Kojima G, Kendrick D, Skelton DA, et al. Frailty predicts short-term incidence of future falls among British community dwelling older people: a prospective cohort study nested within a randomised controlled trial. BMC Geriatrics 2015;15:155.

34. Moreland JD, Richardson JA, Goldsmith CH, Clase CM. Muscle weakness and falls in older adults: a systematic review and meta-analysis. J Am Geriatr Soc 2014;52(7):1121-1129.

35. Fourteau M, Virecoulon Giudici K, Rolland Y, et al. Associations between multidomain lifestyle interventions and intrinsic capacity domains during aging: a narrative review. J Aging Res \& Lifestyle 2020;9:16-25.

36. Hortoba'gyi T, Mizelle C, Beam S, DeVita P. Older adults perform activities of daily living near their maximal capabilities. J Gerontol Biol Sci Med Sci 2003;58A(5):453-460.

37. Ahlund K, Ekerstad N, Back M, Karlson B, Oberg B. Preserved physical fitness is associated with lower 1-year mortality in frail elderly patients with severe comorbidity burden. Clinical Interventions in Aging 2019;14:577-586. 